medRxiv preprint doi: https://doi.org/10.1101/2020.05.18.20105882; this version posted May 22, 2020. The copyright holder for this preprint (which was not certified by peer review) is the author/funder, who has granted medRxiv a license to display the preprint in perpetuity. It is made available under a CC-BY-ND 4.0 International license .

\title{
Efficient Prevalence Estimation and Population Screening for SARS-CoV-2
}

Kenneth Gundersen $\mathrm{PhD}^{1}$, Jan Terje Kvaløy $\mathrm{PhD}^{2,3 *}$, Håkon K. Gjessing $\mathrm{PhD}^{4,5}$, Iren Høyland Löhr $\mathrm{PhD}^{6}$

${ }^{1}$ Lyngvegen 5, 4340 Bryne, Norway

${ }^{2}$ Department of Mathematics and Physics, University of Stavanger, 4036 Stavanger, Norway

${ }^{3}$ Department of Research, Stavanger University Hospital, 4068 Stavanger, Norway

${ }^{4}$ Centre for Fertility and Health, Norwegian Institute of Public Health, 0213 Oslo, Norway

${ }^{5}$ Department of Global Public Health and Primary Care, University of Bergen, 5020 Bergen, Norway

${ }^{6}$ Department of Medical Microbiology, Stavanger University Hospital, 4068 Stavanger, Norway

*Corresponding author:

Jan Terje Kvaløy PhD

Department of Mathematics and Physics

University of Stavanger

4036 Stavanger

Norway

E-mail: jan.t.kvaloy@uis.no

Phone: +4751832255

\begin{abstract}
The testing capacity for SARS-CoV-2 using RT-PCR analyses can be substantially increased by using pooling techniques. Here we discuss two important consequences of this. First, prevalence estimation can be done with a very limited number of RT-PCR analysis. Second, for screening purposes, the number of detected cases can largely increased.
\end{abstract}


medRxiv preprint doi: https://doi.org/10.1101/2020.05.18.20105882; this version posted May 22, 2020. The copyright holder for this preprint

(which was not certified by peer review) is the author/funder, who has granted medRxiv a license to display the preprint in perpetuity.

It is made available under a CC-BY-ND 4.0 International license .

\section{Efficient Prevalence Estimation and Population Screening for SARS-CoV-2}

\section{Introduction}

The most common testing method for SARS-CoV-2 is RT-PCR analysis of nasopharyngeal and/or throat swab samples. In most countries, testing capacity is highly limited due to lack of equipment and necessary testing reagents. Consequently, some laboratories have explored pooling techniques to increase the number of individuals tested with the available number of tests. ${ }^{1}$ At Stavanger University Hospital we have operationally verified the possibility of pooling eight samples with maintained diagnostic sensitivity. We would like to draw the attention to two, maybe less obvious, opportunities enabled by pooling of up to 32 or even more samples that appear to be possible within the PCR protocol recommended by WHO for SARS-CoV-2 detection.

\section{SARS-CoV-2 prevalence measurement with minimal number of PCR tests}

Determination of the prevalence of SARS-CoV-2 in the population is of high importance for a number of reasons. For instance, the "true" prevalence is a crucial input to epidemiologic modelling, and for monitoring the effect of social distancing and other measures to suppress spread of SARS-CoV-2. ${ }^{2}$ By single-step pooled analysis, the number of PCR analyses necessary is reduced by a factor equal to the number of samples in each pool. An estimate of the prevalence can be calculated from the proportion of positive pools; it is not necessary to identify the exact number of positive samples in each pool. The technique is well described in the literature ${ }^{3-5}$ and corrects for the possibility that there is more than one positive sample in each positive pool. Details about tools and methods are given in the supplementary material. To further reduce the number of required PCR analyses, a higher degree of pooling can be employed, even if the corresponding reduction in sensitivity would be considered unacceptable for diagnostic purposes. For prevalence estimation the reduced sensitivity is, however, straightforward to correct for, as long as it is known. Yelin et al. ${ }^{1}$ have indicated that with pooling of 32 samples the sensitivity is $90 \%$ (10\% false negative rate), relative to that of single sample analysis. With this degree of pooling it would take 200 PCR tests to estimate the prevalence from a dataset of 6400 individual samples. Figure $\mathrm{S} 1$ in the supplementary material indicates the $90 \%$ confidence intervals for various sample sizes, degree of pooling and prevalences.

Maximizing the number of detected cases when screening in a low prevalence population The most common purpose of pooled testing is to analyze as many samples as possible with a given laboratory capacity and maintain a diagnostic level of sensitivity. Further, to combat the current covid-19 epidemic many countries are adapting a "suppression" strategy as recommended by Ferguson et al. ${ }^{2}$ and WHO. ${ }^{6}$ The basic idea of this strategy is, after a period of aggressive social distancing measures, to do extensive testing, case tracing and isolation to keep the prevalence at a low level until a vaccine becomes available. In this context it is important to maximize the number of infected people detected with the available testing resources, rather than to insist that all tests from the community setting are being performed with the highest level of sensitivity. Currently, the limited literature indicates that with a pool size of 32 the sensitivity drops to around $90 \% .{ }^{1}$ When used for population screening pooling of 32 samples will nevertheless allow for detection of far more infected people than for the same number of PCR tests if using one test per person. If screening a population with a prevalence of 0.005 and using pooling of eight samples a mean of around 6000 people can be 
medRxiv preprint doi: https://doi.org/10.1101/2020.05.18.20105882; this version posted May 22, 2020. The copyright holder for this preprint (which was not certified by peer review) is the author/funder, who has granted medRxiv a license to display the preprint in perpetuity. It is made available under a CC-BY-ND 4.0 International license .

screened and a mean of 30 cases detected using 1000 PCR tests. By pooling 32 samples, however, around 12000 people can be screened and a mean of 55 cases detected with the same 1000 PCR tests. Figure S2 shows the corresponding numbers for a range of prevalence values in the screened population. It appears that if using this approach some high-income countries already would have the PCR capacity needed to perform extensive screening of the public, if the prevalence is first suppressed to a low level with strong social distancing measures.

Limitations of both of the above suggested applications of extended pooling is that while the required number of PCR analysis is reduced, the number of samples to be collected is unchanged. Further, the relationship between pool size and test sensitivity is not yet fully established and might also vary between individual laboratories and various PCR protocols, and therefore have to be verified locally.

\section{Transparency declaration}

The authors have no conflicts to declare. No external funding relevant for this work was received by any of the authors.

\section{References}

1 Yelin I, Aharony N, Shaer-Tamar E, et al. Evaluation of COVID-19 RT-qPCR test in multi-sample pools. medRxiv 2020; published online March 27. DOI:10.1101/2020.03.26.20039438 (preprint).

2 Ferguson NM, Laydon D, Nedjati-Gilani G, et al. Report 9: Impact of non-pharmaceutical interventions (NPIs) to reduce COVID19 mortality and healthcare demand. Imperial College COVID-19 Response Team, 2020, https:/www.imperial.ac.uk/media/imperialcollege/medicine/mrc-gida/2020-03-16-COVID19-Report-9.pdf (accessed March, 20).

3 Speybroeck N, Williams CJ, Lafia KB, Devleesschauwer B, Berkvens D. Estimating the prevalence of infections in vector populations using pools of samples. Med Vet Entomol 2012; 26: 361-71.

4 Liu A, Liu C, Zhang Z, Albert PS. Optimality of group testing in the presence of misclassification. Biometrika 2012; 99: 245-51.

5 Warasi MS, Tebbs JM, McMahan CS, Bilder CR. Estimating the prevalence of multiple diseases from two-stage hierarchical pooling. Statist Med 2016; 35: 3851-64.

6 WHO. Global surveillance for COVID-19 caused by human infection with COVID-19 virus: Interim guidance 20 March 2020. World Health Organization, 2020. https://apps.who.int/iris/handle/10665/331506 (accessed Apr 6, 2020). 


\title{
Efficient Prevalence Estimation and Population Screening for SARS-CoV-2 Supplementary Material
}

\author{
Kenneth Gundersen, Jan Terje Kvaløy, Håkon K. Gjessing and Iren Høyland Löhr
}

This supplementary file first provides the plots referenced in the main text. The calculations behind the plots are explained at the end of the document. The $\mathrm{R}$ code used to generate the plots can be found in the GitHub repository: https://github.com/jtkgithub/SARSCoV2_pooled_testing.

\section{Prevalence estimation}

Figure S1 shows $90 \%$ confidence intervals for the estimated prevalence obtained for different sample sizes (number of persons sampled), degrees of pooling, and prevalences. Figure S3 below shows the sensitivity assumed in these calculations, depending on the degree of pooling.
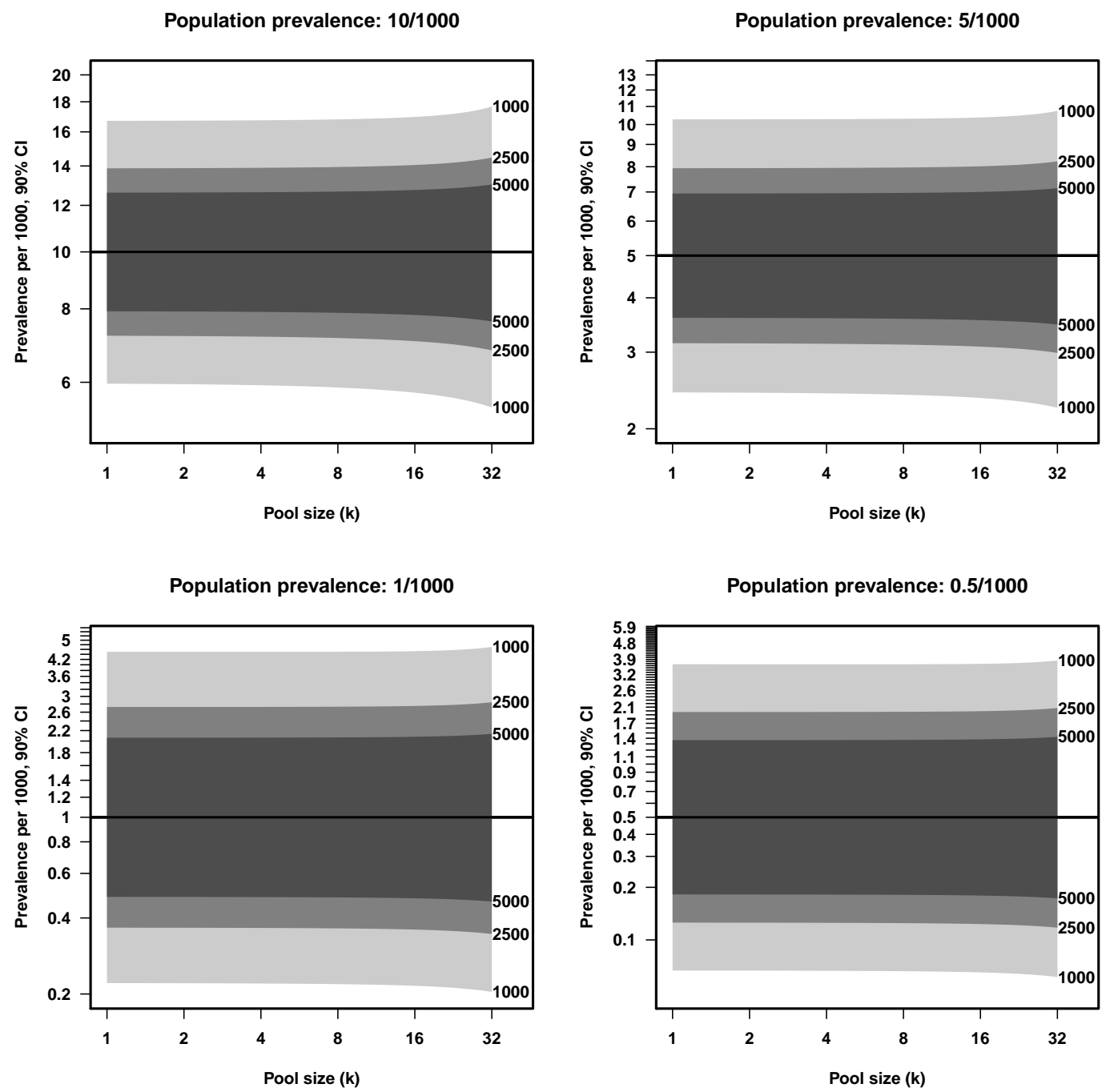

Figure S1: Precision (90\% confidence intervals) of prevalence estimates based on pooled sampling, depending on prevalence, degree of pooling, and the total number of persons sampled (shown in right hand column of the plot). Note that the number of pools needed to be analyzed is the number of persons divided by the degree of pooling. The calculations assume a pool analysis sensitivity as shown in Figure S3. See text for further description. 


\section{Screening}

Figure S2 shows how many persons can be included in the screening and the expected number of infected persons identified for three different screening strategies. The first strategy is to do no pooling. The second strategy is to first test samples in pools of 8 . For the pools that are positive, samples from all 8 persons are tested individually. In the third strategy samples are first tested in pools of 32 . For pools that are positive a second test in 8 pools of size 4 are made. Then finally for the positive pools of 4 , individual tests are run.
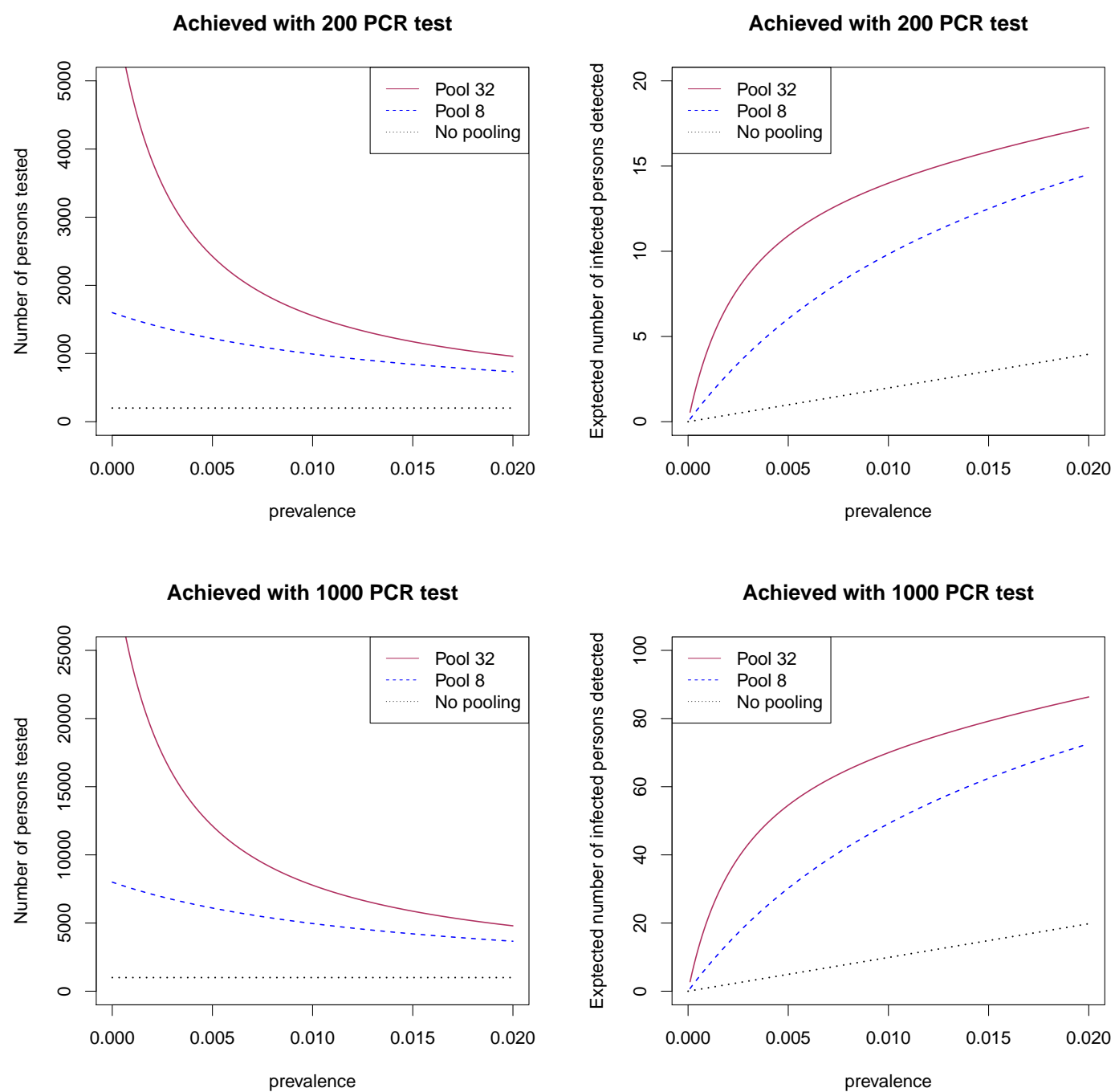

Figure S2: The left plots depicts the number of persons that could be tested and the right plots the expected number of infected persons that would be identified with two different PCR budgets and three different test strategies: 1) No pooling; 2) Pool size 8 in the first testing and re-testing of all samples in the positive pools; 3) Pool size 32 in the first testing, re-testing in pools of 4 for the positive pools, re-testing all for the positive pools of size 4 .

In the illustrations shown in Figure S2 we have assumed a sensitivity of $99 \%$ for pools of size 8 and a sensitivity of $90 \%$ for pools of size 32 . Similar plots for other scenarios can be generated using the R code in the GitHub repository. Note that both the numbers of persons included and the expected number of cases identified scale linearly with the number of available PCR analyses. For 1000 analyses the numbers are five times that of 200 analyses. 


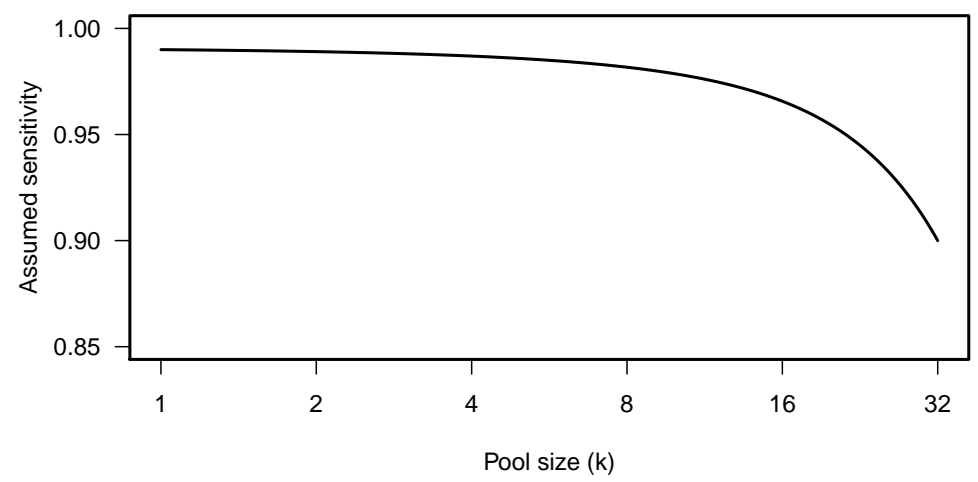

Figure S3: Sensitivity of pooled samples, as assumed in the confidence interval calculations for Figure S1

\section{Mathematical details}

Let $p$ be the true prevalence, $k$ the number of samples merged in one pool and $\pi(k)$ the probability that a pool of $k$ tests is positive. Let further $s(k)$ be the sensitivity of the test for a pool of $k$ tests. We assume the specificity of the test to be 1 . Then

$$
\pi(k)=s(k)\left(1-(1-p)^{k}\right) .
$$

Prevalence estimation For prevalence estimation, $p$, we first estimate $\pi(k)$ by

$$
\hat{\pi}(k)=\frac{N_{+}}{N}
$$

where $N_{+}$is the number of positive pools and $N$ is the number of pools. The total number of persons tested is $n_{\text {pers }}=k N$. From (1) and (2) we get that

$$
\hat{p}=1-\left(1-\frac{\hat{\pi}(k)}{s(k)}\right)^{1 / k}=1-\left(1-\frac{N_{+}}{s(k) N}\right)^{1 / k} .
$$

Moreover, to obtain a confidence interval for $\hat{p}$ we propose to first calculate Wilson confidence intervals ${ }^{1}$ for $\hat{\pi}(k)$ and map this to confidence limits for $\hat{p}$ by inserting the upper and lower limits in (3). Wilson confidence intervals are available from the binom.confint function in the $\mathrm{R}$ package binom ${ }^{2,3}$. Additional statistical tools for computing prevalences from pooled samples can be found in the prevalence package ${ }^{4,5}$.

Screening strategies For considerations around the screening strategies let first $N_{P C R}$ be the total number of PCR analyses which needs to be performed and let $N_{P C R, 1}$ be the number of PCR analyses run in the first round. With no pooling $N_{P C R}=N_{P C R, 1}$. For pooling stratgies $N_{P C R}$ will be a random variable since we do not know how many positive pools we will find. For the strategy with pooling of 8 samples we have

$$
\mathrm{E}\left(N_{P C R}\right)=N_{P C R, 1}+N_{P C R, 1} \cdot \pi(8) 8 \quad \Rightarrow \quad N_{P C R, 1}=\frac{\mathrm{E}\left(N_{P C R}\right)}{1+\pi(8) 8} .
$$

By inserting the budget of available PCR analyses for $\mathrm{E}\left(N_{P C R}\right)$ (e.g. 200 and 1000 in Figure S2) we get that the total number of persons that can be tested is $n_{\text {pers }}=8 N_{P C R, 1}$. Let $n_{\text {pos }}$ be the total number of identified positive cases, and let $n_{p o s, 8}$ denote the number of positive cases in a pool of 8 . Then

$$
\mathrm{E}\left(n_{p o s}\right)=N_{P C R, 1} \cdot \pi(8) \mathrm{E}\left(n_{p o s, 8} \mid n_{p o s, 8} \geq 1\right)=\frac{N_{P C R, 1} \cdot \pi(8) 8 p}{(1-p)^{8}} .
$$


For the strategy starting with 32 pools then re-testing in pools of 4 if positive and finally testing all samples in positive pools of 4 , similar calculations give.

$$
N_{P C R, 1}=\frac{\mathrm{E}\left(N_{P C R}\right)}{1+\pi(32)\left(8+4 \mathrm{E}\left(X \mid n_{p o s, 32} \geq 1\right)\right)} .
$$

Here $X$ is the number of positive pools among eight pools of size 4 , and $\mathrm{E}\left(X \mid n_{\text {pos }, 32} \geq 1\right)$ can be approximated by

$$
\begin{aligned}
\mathrm{E}\left(X \mid n_{\text {pos }, 32} \geq 1\right)= & {\left[P\left(n_{\text {pos }, 32}=1\right)+\left(\frac{3}{31}+2 \frac{28}{31}\right) P\left(n_{\text {pos }, 32}=2\right)\right.} \\
& +\left(\frac{3}{31} \frac{2}{30}+2\left(\frac{3}{31} \frac{28}{30}+\frac{28}{31} \frac{6}{30}\right)+3 \frac{28}{31} \frac{24}{30}\right) P\left(n_{\text {pos }, 32}=3\right) \\
& \left.+\sum_{k=4}^{7} k P\left(n_{\text {pos }, 32}=k\right)+8 P\left(n_{\text {pos }, 32} \geq 8\right)\right] / P\left(n_{\text {pos }, 32} \geq 1\right) .
\end{aligned}
$$

The last two terms $\sum_{k=4}^{7} k P\left(n_{\text {pos }, 32}=k\right)+8 P\left(n_{\text {pos }, 32} \geq 8\right)$ implies that this is an upper bound, the approximation being very accurate for small $p$. Finally, we get the expected number of detections by

$$
\mathrm{E}\left(n_{p o s}\right)=N_{P C R, 1} \cdot \pi(32) \mathrm{E}\left(n_{p o s, 32} \mid n_{p o s, 32} \geq 1\right)=\frac{N_{P C R, 1} \cdot \pi(32) 32 p}{(1-p)^{32}} .
$$

\section{Acknowledgements}

We thank Eva Bernhoff and Ola Brønstad Brynildsrud for useful discussions and contributions.

\section{References}

1 Agresti A, Coull BA. Approximate is Better than "Exact" for Interval Estimation of Binomial Proportions. The American Statistician. 1998;52(2):119-126.

2 R Core Team. R: A Language and Environment for Statistical Computing. Vienna, Austria; 2019.

3 Dorai-Raj S. binom: Binomial Confidence Intervals For Several Parameterizations; 2014. R package version 1.1-1.

4 Devleesschauwer B, Torgerson P, Charlier J, Levecke B, Praet N, Roelandt S, et al.. prevalence: Tools for prevalence assessment studies.; 2015. R package version 0.4.0.

5 Speybroeck N, Williams CJ, Lafia KB, Devleesschauwer B, Berkvens D. Estimating the prevalence of infections in vector populations using pools of samples. Medical and Veterinary Entomology. 2012 Dec;26(4):361-371. 